\title{
Lessons Learned from the First Decade of Laser- Assisted Drug Delivery
}

Tamara Searle $\cdot$ Faisal R. Ali $\cdot$ Firas Al-Niaimi

Received: November 8, 2020 / Published online: January 19, 2021

(c) The Author(s) 2021

\section{ABSTRACT}

Laser-assisted drug delivery augments the distribution and penetration of topically applied treatments, leading to enhanced delivery and bioavailability. We discuss the therapeutic application of laser-assisted drug delivery in clinical practice in cases of non-melanoma skin cancer, vitiligo, melasma, scarring, and alopecia (female pattern hair loss, male pattern hair loss, alopecia areata) as well as for vaccination, local anaesthesia, analgesia, viral warts, infantile

T. Searle

University of Birmingham, Birmingham, UK

F. R. Ali · F. Al-Niaimi

St John's Institute of Dermatology, Guy's and St

Thomas' NHS Foundation Trust, London, UK

F. R. Ali

Vernova Healthcare CIC, Macclesfield, UK

F. Al-Niaimi $(\bowtie)$

Department of Dermatology, Aalborg University

Hospital, Aalborg, Denmark

e-mail: firas55@hotmail.com haemangiomas and cosmetic uses, and we review clinical studies that have used this technique over the last decade. Our review shows that the application of laser-assisted drug delivery enhances topical agent efficacy, potentially reducing the agent concentration and duration of topical treatment required. Future research into the use of laser-assisted drug delivery before topical therapies is needed to establish the optimal techniques to enhance drug delivery and thus improve patient outcomes.

Keywords: Analgesia; Female pattern hair loss; Infantile haemangiomas; Laser; Local anaesthesia; Non-melanoma skin cancer; Scarring; Vaccination; Viral warts; Vitiligo 


\section{Key Summary Points}

Laser-assisted drug delivery augments the distribution and penetration of topically applied treatments, leading to enhanced delivery and bioavailability.

Laser-assisted drug delivery is used therapeutically in clinical practice in cases of non-melanoma skin cancer, vitiligo, melasma, scarring, alopecia (female pattern hair loss, male pattern hair loss, alopecia areata) as well as for vaccination, local anaesthesia, analgesia, viral warts, infantile haemangiomas and cosmetic uses.

We found that laser-assisted drug delivery enhanced the efficacy of topical agents, potentially reducing the agent concentration and duration of topical treatment required.

Future research into the use of laserassisted drug delivery before topical therapies is needed to establish the optimal techniques to enhance drug delivery and thus improve patient outcomes.

\section{DIGITAL FEATURES}

This article is published with digital features to facilitate understanding of the article. You can access the digital features on the article's associated Figshare page. To view digital features for this article go to https://doi.org/10.6084/m9. figshare.13378829.

\section{INTRODUCTION}

Topical medications undergo limited epidermal absorption due the robustness of the skin barrier. Laser-assisted drug delivery (LADD) involves the use of an ablative fractional laser, namely a carbon dioxide $\left(\mathrm{CO}_{2}\right)$ or erbium- doped yttrium aluminium garnet (Er:YAG) laser, to create microscopic channels that allow drugs to travel through the epidermis $[1,2]$. Er:YAG and $\mathrm{CO}_{2}$ lasers are both ablative, but with different ablation-to-coagulation ratios depending on the biological interaction. Er:YAG laser light has a much higher absorption coefficient in water than $\mathrm{CO}_{2}$ laser light, leading to a higher ablation-to-coagulation ratio and a shallower depth of penetration, although this also depends on the laser fluence and beam spot size. While both lasers are equally effective at providing a path for therapeutic agents through the stratum corneum, the $\mathrm{CO}_{2}$ laser, with its deeper beam profile and coagulative zone, is preferable to the Er:YAG laser for more penetrative beam dermal drug delivery.

Ablative laser devices have been utilised in patients with a range of conditions to facilitate drug delivery through skin layers. This technique enables high molecular weight molecules to pass through the stratum corneum. Ablative fractional laser technology (AFXL) works through photothermolysis: tissue is destroyed in multiple skin layers, starting with the stratum corneum. The depth of the tissue that is destroyed by the laser depends on the laser parameters employed. The resulting channel through the skin is encapsulated by a microscopic treatment zone that enables the penetration of topical molecules into the skin layer to be treated, resulting in faster healing with less scarring [3].

This article focuses on the use of ablative laser devices to enhance the delivery of drugs that are used to treat a variety of conditions. The most recent advances in this field are reviewed. The article only considers previously conducted studies; it does not discuss any new studies with human participants or animals performed by any of the authors.

\section{Non-melanoma Skin Cancers and Skin Cancers}

\section{Actinic Keratoses}

Actinic keratoses (AKs) are premalignant lesions that can be considered part of the non-melanoma skin cancer (NMSC) family $[5,6]$. 
Treatment options for AKs include cryotherapy, curettage, excision, photodynamic therapy (PDT), topical 5-fluorouracil, and imiquimod. A recent systematic review noted that when AFLX-assisted PDT was employed to treat AK, the 12-month AK clearance rate increased from $45-51 \%$ (for non-AFXL-assisted PDT) to $78-85 \%$ [7].

PDT monotherapy was compared with AFXL pretreatment followed by PDT $(10 \mathrm{~mJ}$ per pulse, $0.12 \mathrm{~mm}$ spot, $5 \%$ density) in a trial of 15 patients with AKs. After three months of followup, the AFXL-PDT therapy was found to be more efficacious than PDT monotherapy, producing a complete lesion response in $88 \%$ of patients, as compared to $59 \%$ for PDT monotherapy. Nevertheless, side effects such as erythema, crusting, pigmentation and pain occurred more frequently in patients who received the AFXL-PDT treatment than in those who underwent the PDT monotherapy. This study suggests that although AFXL augments the penetration of PDT, side effects are more pronounced when this pretreatment is applied [8].

Patients who receive organ donations are at greater risk of AKs and other NMSCs, and the resulting lesions are less responsive to PDT [6]. AFXL-PDT $(30 \mathrm{~W}, 0.12 \mathrm{~mm}$ spot size, two passes) was compared with AFXL alone for the treatment of ten transplant patients with AKs. $73 \%$ of the AFXL-PDT group had a complete response, compared with $31 \%$ of the AFXL monotherapy group [9].

In a separate study of 46 patients, 22 received PDT whilst 24 underwent AFXL-PDT. Both groups were then treated with the photosensitiser methyl aminolevulinate (MAL; incubation for $90 \mathrm{~min}$ ). Clearance rates at 10 weeks were $64.7 \%$ in the PDT-only group and $71.4 \%$ in the AFXL-PDT group, which was not indicative of a significant difference in clearance between the groups. This study suggests that AFXL pretreatment could reduce the incubation time needed for the photosensitiser without affecting efficacy [10].

A recent case report presented two patients with multiple AKs who received single sessions of Er:YAG laser ablation (Etherea, Vydence Medical Sao Paulo, Brazil; wavelength:
$2940 \mathrm{~nm}$, spot size: $8 \mathrm{~mm}$, density: $100 \mathrm{MTX} /$ $\mathrm{cm}^{2}$, single mode $(500 \mu \mathrm{s})$, energy: $12.5 \mathrm{~mJ} /$ MTZ). This treatment was followed by a combination of 5\% 5-FU and 2\% alpha-bisabolol for six nights. The patients presented excellent clinical responses, with complete lesion clearance and improved skin quality, and this response was maintained at nine months of follow-up [11].

These studies suggest that pretreatment with AFXL could enhance the penetration of topical therapies such as 5-FU or imiquimod that are used to treat AKs and other NMSCs. It could also reduce the number of therapeutic sessions required, which would be particularly helpful for patients who are less compliant or have multiple difficult-to-reach lesions [12].

\section{Bowen's Disease}

Bowen's disease is a type of squamous cell carcinoma in situ in which there is full-thickness dysplasia of the epidermis. If left untreated, Bowen's disease can progress into squamous cell carcinoma (SCC). AFXL pretreatment of Bowen's disease preceding MAL-PDT has been shown to shorten the photosensitiser incubation time required to achieve the same efficiency (70 min compared to the typical $180 \mathrm{~min})$ [13].

A systematic review investigating AFXL-assisted treatments for keratinocyte carcinoma and its precursors found that the 12-month clearance rate of Bowen's disease increased from $50-55 \%$ to $88 \%$ with AFXL-assisted therapy. [7]

A randomised controlled trial of 21 patients with multiple Bowen's disease lesions had a clearance rate of $93.8 \%$ three months after a single AFXL-PDT treatment, compared with $73.1 \%$ three months after the first of two sessions of MAL-PDT. The 12-month relapse rate was also significantly lower for AFXL-PDT (6.7\%) than for MAL-PDT (31.6\%) [14].

In a separate case series of 28 patients with Bowen's disease, patients were treated with a single session of $\mathrm{CO}_{2}$-AFXL $(0.12 \mathrm{~mm}$ spot size, $10 \mathrm{~mJ}$ per pulse, single pulse, $5 \%$ density) followed by one application of topical 5\% 5-FU under occlusion for 7 days. It was reported that $100 \%$ of the Bowen's disease lesions had cleared histologically after 8 weeks [15]. 


\section{Basal Cell Carcinoma}

PDT therapy is not typically recommended for nodular BCCs because penetration is often limited by the thickness of the tumour itself [16]. Pretreatment with AFXL is hypothesised to create channels for a photosensitising agent to reach the tumour [2].

A case series of three patients with nodular BCCs were treated with AFXL prior to MAL-PDT treatment. Two patients presented complete clinical and histological responses; the third had a partial response, probably because that patient had a sclerosing tumour subtype for which PDT is less effective. Randomised controlled trials are needed to investigate the use of AFXL in the pretreatment of BCC [17].

A separate study investigated nodular BCCs treated with a 980-nm gallium arsenide diode laser under ultrasound control $(n=56)$ [18]. After 3 weeks, each BCC was divided in half, with one half receiving $\mathrm{CO}_{2}$-AFXL and the other half receiving curettage followed by ALA-PDT therapy. Clearance rates (confirmed histologically) were greater for the halves treated with AFXL (93\%) than for the halves that underwent curettage (80\%).

In another study, 32 patients were randomised to receive AFXL-PDT or conventional MAL-PDT for the treatment of histologically confirmed high-risk nodular BCCs [19]. A 100\% cure rate was achieved at 3 months when a $\mathrm{CO}_{2^{-}}$ AFXL-PDT (5\% density, $80 \mathrm{~mJ}, 1000 \mu \mathrm{m}$ ablation depth) treatment was used, as compared with an $88 \%$ cure rate for those treated with conventional PDT. Nevertheless, the histological tumour clearance rates for both therapies after 12 months were comparable.

\section{Actinic Cheilitis}

Patients with actinic cheilitis $(n=33)$ were randomised to receive either one session of AFXL-PDT or two sessions of MAL-PDT [20]. $92 \%$ of those treated with AFXL-PDT showed a complete response after three and twelve months, as compared with $59 \%$ of those treated with MAL-PDT. There was no significant difference between the side-effect profiles for the two treatments. This study supports the possible use of AFXL with PDT in cases of actinic cheilitis. PDT is not usually as effective for actinic cheilitis as it is for AKs, although both conditions present a similar risk of subsequent squamous cell carcinoma development [21].

\section{Pigmentary Disorders}

\section{Vitiligo}

Recalcitrant vitiligo patients $(n=25)$ were treated on one side of the body with $\mathrm{CO}_{2}$-AFXL (every 2 weeks) followed by betamethasone topical solution under occlusion followed by narrowband-ultraviolet $\mathrm{B}$ (NB-UVB) phototherapy (2-3 times a week for 6 months), and on the other (control) side of the body with $\mathrm{CO}_{2}$-AFXL and NB-UVB alone [22]. Over 50\% repigmentation occurred in $44 \%$ of the betamethasonetreated sides, which was significantly greater than the corresponding rate for the control sides, due to greater corticosteroid penetration. However, the length of treatment and the significant cost may make this treatment course less suitable for parsimonious healthcare systems [22].

\section{Melasma}

Tranexamic acid has been used for a wide variety of skin conditions, including melasma [23]. A split-face, double-blind, randomised controlled study of 46 melasma patients treated with a 1927-nm thulium laser on both sides of the face and with tranexamic acid on one side of the face was reported. Significant improvements were observed on both face sides, and there was no statistically significant difference in improvement between the sides [24]. Similar findings were reported for a randomized comparative split-face study of 30 melasma patients treated with a fractional ablative $\mathrm{CO}_{2}$ laser alone or in combination with tranexamic acid [25]. Both the combination therapy and the monotherapy resulted in a significant reduction in the Melasma Area and Severity Index (MASI). Patients reported no difference in improvement between the face sides, but the degree of improvement in the MASI score was greater for the side that was treated with the fractional ablative $\mathrm{CO}_{2}$ laser only. The use of tranexamic acid in combination with fractional ablative $\mathrm{CO}_{2}$ laser treatment requires further 
investigation [25]. A separate study compared microneedling on one side of the face with fractional $\mathrm{CO}_{2}$ laser treatment in combination with tranexamic acid on the other side of the face in 30 melasma patients, and found no statistically significant difference between the sides [26]. A randomized, single-blinded, splitface clinical trial of 40 melasma patients treated with topical hydroquinone $4 \%$ or a combination of topical hydroquinone $4 \%$ and a fractional laser noted improvements in hyperpigmentation and darkness on both face sides, with no significant difference in improvement between sides [27]. The use of an Er:YAG laser to enhance topical hydroquinone penetration was investigated in 30 melasma patients [28]. In a split-face study, patients were treated on one side with six sessions involving the application of a fractional Er:YAG laser and then $4 \%$ hydroquinone cream, with a 2 -week interval between each session, and on the other side with the $4 \%$ hydroquinone cream alone. Pretreatment with the Er:YAG laser led to significantly better results (much larger reductions in the MASI) than using hydroquinone alone [28]. A recent split-face study of 22 patients with melasma compared the effects of a fractional Er:YAG laser combined with a topical steroid to the effects of fractional Er:YAG monotherapy [29]. Significantly better outcomes were achieved with the combined therapy, with the MASI score decreasing much more than in the Er:YAG monotherapy group [29].

\section{Anaesthesia and Analgesia}

\section{Local Anaesthetics}

Many dermatological surgical procedures require a local anaesthetic. Topical anaesthetic agents may not penetrate deeply enough, whilst injections are associated with discomfort [2].

The application of an Er:YAG laser prior to the use of topical $4 \%$ lidocaine was found to reduce the pain associated with injection in as little as 5 min when compared to the application of the laser and placebo (reduction of $62 \%$ ) or lidocaine monotherapy (61\% reduction) [30]. A randomised controlled trial of 61 patients who underwent cannulation in A\&E found that pain levels were significantly lower when a Er:YAG laser was used before topical $4 \%$ lidocaine [31].

These findings were replicated by Meesters and colleagues, who found that pretreatment of ten patients with $\mathrm{CO}_{2}$-AFXL $(5 \%$ density, $2.5 \mathrm{~mJ} /$ microbeam) prior to the application of topical local anaesthetics such as articaine hydrochloride $40 \mathrm{mg} / \mathrm{ml}$, adrenaline $10 \mu \mathrm{g} / \mathrm{ml}$ solution (AHES) and lidocaine $25 \mathrm{mg} /$ g with prilocaine $25 \mathrm{mg} / \mathrm{g}$ cream $\quad$ EMLA $^{\circledR}$ cream) was painless [32]. Self-reported pain levels due to a painful stimulus were significantly lower following the application of AFXL and these topical local anaesthetics than following a sham laser therapy. AHES plus AFXL was found to be the most efficacious anaesthetic method, likely due to the liquidity of this treatment option [32].

In a randomised controlled trial, twelve patients were treated on one side of the face with a low-fluence Er:YAG laser prior to the application of $5 \%$ topical lidocaine and wholeface resurfacing in two sessions [33]. Self-reported pain scores were significantly less for the side pretreated with the Er:YAG laser, although only $56 \%$ of patients were able to tolerate the second resurfacing treatment [33].

\section{Analgesia}

The effect of AFXL on diclofenac penetration was investigated by Bachhav and colleagues. The permeation of diclofenac depended on the pore frequency and the laser fluence used. Clinically, the results of this study indicate that AFXL pretreatment of the skin is an effective method of augmenting the local absorption of topical diclofenac while avoiding systemic side effects such as gastric complications and renal problems [22].

Similarly, systemic side effects of opioids could be avoided by applying topical agents in conjunction with Er:YAG laser pretreatment. This has only been investigated in animal studies, so human trials are required to examine its potential for clinical use [30]. 


\section{Scarring}

AFXL treatment can remove sections of fibrotic scars, normalising collagen structure and composition and improving overall scar appearance [34], and is applicable to keloids [35] and hypertrophic scars[ 36].

In one study, 15 patients with hypertrophic scars received up to five treatments with $\mathrm{CO}_{2}$ AFXL (10-15\% density) followed by topical triamcinolone $(10$ or $20 \mathrm{mg} / \mathrm{ml})$ [36]. After 6 months, improvements in texture, hypertrophy and dyschromia were noted. AFXL treatment permitted greater uniformity of the depth and distribution of triamcinolone and bypassed the pain often associated with intralesional injections [36]. In a prospective double-blinded study of 20 patients with hypertrophic scars, the patients had three treatment sessions every month with $\mathrm{CO}_{2}$-AFXL followed by postoperative treatment with either triamcinolone acetonide $(20 \mathrm{mg} / \mathrm{mL})$ or topical 5-fluorouracil $(50 \mathrm{mg} / \mathrm{mL})$. There were no statistically significant differences between these treatments, but treatment of the scars with 5-fluorouracil did not result in the side effects associated with corticosteroid therapy, such as dermal atrophy and telangiectasia [37].

A double-blind, randomised, split-face trial investigated postoperative wound healing. Fifteen patients were treated with a vitamin C, E and ferulic acid serum on one side of the face and a vehicle cream on the other side immediately after the application of a fractional ablative $\mathrm{CO}_{2}$ laser. Decreased postoperative oedema and erythema were noted for the face sides treated with the laser, as well as significantly increased expression of basic fibroblast growth factor, indicating that wound healing was more rapid with this treatment [38].

In a separate study, 23 patients with 70 keloid scars were treated with Er:YAG-AFXL $\left(180 \mathrm{~J} / \mathrm{cm}^{2}, 5 \%\right.$ coverage) every other week and concomitant betamethasone cream twice daily under occlusion until the scar was flattened or no further improvement was observed [39]. After a median treatment time of nine sessions, the patients showed a median 50\% improvement in scar appearance, and recurrence was $22 \%$ eight months following treatment [39].
Ten patients with keloid scars were treated with an ablative Er:YAG laser (four treatments, 6 weeks between treatments) and then received either an intralesional steroid injection or topical corticosteroids under occlusion for $3 \mathrm{~h}$ [40]. The combination of ablative fractional Er:YAG laser treatment and topical corticosteroids resulted in greater decreases in the mean keloid Vancouver Scar Scale score and the mean pain score than in the corticosteroid injection group [40].

A patient (Fitzpatrick VI) with a ten-year history of keloid was treated with eight sessions of $\mathrm{CO}_{2}$-AFXL immediately followed by laser-assisted drug delivery of a topical triamcinolone acetonide ointment. Excellent cosmetic results were noted at the 22-month follow-up [41]. This case emphasises the safety of AFXL in patients with dark skin when it is used in the correct setting [41].

Nineteen patients with atrophic scars were treated with $\mathrm{CO}_{2}$-AFXL (spot size $120 \mu \mathrm{m}$, depth $375-500 \mu \mathrm{m}$, density $10 \%$ ) followed by the topical application of poly-L-lactic acid (PLLA; Sculptra ${ }^{\circledR}$ ), which is often used as a filler due to its proposed ability to stimulate fibroblast proliferation [42]. After 3 months, improvements in scar contours, atrophy and colour were reported, with proposed mechanisms of action including enhanced PLLA dermal penetration [42].

\section{Cosmetic Uses}

A split-face study investigated 14 patients with signs of photoageing [43]. Both sides of the face were first pretreated with $\mathrm{CO}_{2}$-AFXL. After that, PixelTreatSR serum (Alma Lasers, Caesarea, Israel) was applied to one half of the face, immediately followed by ultrasound pulses to stimulate transepidermal passage. The serum was composed of keratolytics, lipids, vitamins and bioactive peptides. At six months, improvements in pigmentation, fine lines and wrinkles, and overall ageing on the side that received the serum were noted. It is unclear whether this improvement was due to the ultrasound or the serum, but the results of this study support the use of AFXL-assisted cosmeceuticals in cosmetic dermatology [43]. 
A separate split-face study of ten patients investigated the use of $\mathrm{CO}_{2}$-AFXL in combination with topical botulinum neurotoxin type $\mathrm{A}$ (BoNTA) on one side of the face and a saline control on the other side [44]. The side with BoNTA showed significant reductions in periorbital wrinkles at one week and one month post-treatment, suggesting that the delivery of BoNTA could be augmented by AFXL pretreatment. This method of delivering BoNTA could potentially avoid the pain, discomfort, erythema and infection risk associated with BoNTA injections [44]. Future studies comparing BoNTA injections with different topical BoNTA compositions must be carried out to explore the potential use of AFXL prior to BoNTA treatment [45].

\section{Other Uses}

\section{Alopecia}

A study investigated the use of a nonablative erbium glass fractional laser (eight sessions at $7 \mathrm{~mJ}, 3-9 \%$ coverage, density $120 \mathrm{MZT} / \mathrm{cm}^{2}$ ) followed by topical finasteride $0.05 \%$ and growth factors (basic fibroblast growth factor, insulin-like growth factor, vascular endothelial growth factor, and copper peptide 1\%) in four patients with androgenetic alopecia [46]. Improved hair regrowth and density were observed in all patients, with no significant reported side effects [46]. Ablative laser therapy could allow topical agents to be effectively delivered to and distributed uniformly in the dermis, where the majority of the hair follicles are located [47]. A fractional $\mathrm{CO}_{2}$ laser was applied along with a topical triamcinolone spray $(10 \mathrm{mg} / \mathrm{mL})$ to ten patients with recalcitrant alopecia areata. Seven of the eight patients who finished the treatment experienced complete recovery of the area treated [48]. Forty-five male patients with androgenetic alopecia were treated with an ablative fractional $\mathrm{CO}_{2}$ laser and minoxidil combination, the fractional $\mathrm{CO}_{2}$ laser alone, or minoxidil alone. The application of the ablative fractional $\mathrm{CO}_{2}$ laser alone or in combination with minoxidil produced the best results [49].

\section{Amyloidosis}

The effect of AFXL treatment on macular amyloidosis was compared with the effect of AFXLassisted drug delivery of topical steroids and vitamin $C$ in ten patients with cutaneous macular amyloidosis, a type of primary localised cutaneous amyloidosis. Patients underwent four sessions of fractional $\mathrm{CO}_{2}$ laser treatment (power $18 \mathrm{~W}$, spacing $800 \mu \mathrm{m}$, dwell time $600 \mu \mathrm{s}$, stacking 3), with a 4-week interval between sessions [50]. All targeted areas showed significantly decreased pigmentation scores, an improvement in lichenification, and decreased histological amyloid after treatment. The area treated with the fractional laser and corticosteroids demonstrated the largest decrease in amyloid, highlighting the benefits of a fractional $\mathrm{CO}_{2}$ laser in assisting the delivery of topical steroids and topical vitamin C [50].

\section{Vaccination}

Intradermal delivery of vaccines can be more efficacious than the conventional intramuscular route due to the abundance of antigen-presenting cells in the skin [51].

The use of AFXL (5-15\% coverage) to enhance the transcutaneous delivery of a model antigen (ovalbumin, OVA), provoking an immune response, has so far only been demonstrated in animal studies. Further studies of this response in humans are warranted, in particular to address possible safety concerns [51].

\section{Haemangiomas}

The utilisation of topical timolol for the treatment of infantile haemangiomas (IHs) was investigated in a study of nine children [52]. $\mathrm{CO}_{2}$-AFXL was applied weekly to the skin surfaces of deep IHs $(25-30 \mathrm{~mJ} /$ pulse, 5\% density, single pulse), followed by a topical timolol maleate solution (0.5\%) under occlusion five times a day for 14 weeks. $44 \%$ of patients had an excellent response to this treatment, $44 \%$ had a good response, and $11 \%$ had a moderate response, measured via the haemangioma activity score. Treatments were well tolerated, with no reported side effects. This study's findings suggest that the application of AFXL 
pretreatment preceding topical timolol could be an alternative for infants in which systemic therapies are contraindicated, or for localised IHs [52].

A recent study replicated these findings; it investigated the treatment of 30 deep IHs with a fractional Er:YAG laser applied at two-week intervals and subsequent topical timolol maleate $0.5 \%$ applied under occlusion for $30 \mathrm{~min}$ four times a day for 24 weeks [53]. $76.7 \%$ of patients showed excellent regression, $13.3 \%$ had good regression, and $10 \%$ showed moderate regression [53].

\section{Viral Warts}

A Korean study investigated 12 patients with 40 periungual warts treated with $\mathrm{CO}_{2}$-AFXL prior to the application of PDT and MAL as well as red light illumination 180 min later. Patients had an average of 2.2 treatments per wart, with $90 \%$ of the lesions presenting complete clinical clearance and 5\% exhibiting no apparent therapeutic response [54]. A separate study investigated the use of an Er:YAG ablative fractional laser and topical cidofovir for the treatment of recalcitrant plantar warts in two patients. Both patients experienced a significant reduction in lesion size and an improvement in symptoms [55].

\section{Primary Plantar Hyperhidrosis}

Botulinum toxin type A (BTX-A) was used in three patients with primary palmar hyperhidrosis. Immediately afterwards, a fractional $\mathrm{CO}_{2}$ laser was applied; the resulting enhanced delivery of BTX-A led to a decrease in sweat production [56].

\section{DISCUSSION}

Large, robust randomised controlled trials are required to demonstrate the benefit of AXFL when used as a pretreatment for the conditions described above, as well as to investigate any possible associated side effects [57]. Demographic investigations, including those focusing on age, gender and ethnicity, are also warranted to assess the benefits of AXFL in different patient subgroups. Laser parameters such as the fluence, density, number of treatments and treatment length must be optimised to maximise drug penetration and minimise skin damage [2].

The literature supports the laser-assisted delivery of anti-inflammatory, antineoplastic and antioxidative agents to treat scarring, photoageing and neoplasias [58]. Clinicians face challenges such as unpredictable dosing and therapeutic responses, the possibility of local and systemic reactions, and inconsistent treatment regimes [58].

The vehicle of choice, such as a gel, patch, cream or ointment, as well as the length of treatment, the frequency of use and the concentration of the drug must also be considered [2]. Any side effects resulting from the increased penetration of drugs designed for topical application following the application of laser-assisted delivery must not be overlooked, and care must be taken when investigating this issue $[2,4]$. A cost-benefit analysis of AFXL pretreatment must also be performed, and the efficacy of this pretreatment must be substantiated before its use over preestablished treatments can be warranted [2].

Based on relevant studies, it appears that the most important step in the treatment of most superficially located pathologies is traversing the stratum corneum, that the difference in wavelength between $\mathrm{CO}_{2}$ and Er:YAG lasers is not particularly significant, and that a relatively low laser density is as effective for laser-assisted delivery as a larger density. The depth of ablation achieved with both lasers depends on the device characteristics, such as the beam profile and fluence used. Deeper dermal pathologies require a channel that reaches the required depth, which necessitates a higher laser fluence. It should be noted that the literature in this field includes more studies of the use of a $\mathrm{CO}_{2}$ rather than an Er:YAG laser due to the deeper penetration profiles of currently available $\mathrm{CO}_{2}$ laser devices, as well as the possible added advantage that the surrounding coagulative zone acts as a slow-release reservoir for drug release. 


\section{CONCLUSIONS}

Overall, current research suggests that AFXL pretreatment enhances the penetration and absorption of topical agents. The strongest evidence of a benefit of AFXL pretreatment is provided by the application of MAL-PFT to AKs, which produces long-lasting results compared with conventional PDT and no adverse effects [59]. Therefore, the addition of AFXL pretreatment to current therapies could make them more efficacious, meaning that treatment lengths could be shortened and active agent concentrations could be weakened, reducing the potential for side effects [60]. Future studies involving larger patient cohorts will aim to improve our understanding of this pretreatment option and to optimise its parameters to achieve the best possible patient outcomes.

\section{ACKNOWLEDGEMENTS}

Funding. No funding or sponsorship was received for this study or the publication of this article.

Authorship. All named authors meet the International Committee of Medical Journal Editors (ICMJE) criteria for authorship for this article, take responsibility for the integrity of the work as a whole, and have given their approval for this version to be published.

Disclosures. Tamara Searle, Faisal R. Ali and Firas Al-Niaimi have nothing to disclose.

Compliance with Ethics Guidelines. This article is based on previously conducted studies and does not contain any new studies with human participants or animals performed by any of the authors.

Open Access. This article is licensed under a Creative Commons Attribution-NonCommercial 4.0 International License, which permits any non-commercial use, sharing, adaptation, distribution and reproduction in any medium or format, as long as you give appropriate credit to the original author(s) and the source, provide a link to the Creative Commons licence, and indicate if changes were made. The images or other third party material in this article are included in the article's Creative Commons licence, unless indicated otherwise in a credit line to the material. If material is not included in the article's Creative Commons licence and your intended use is not permitted by statutory regulation or exceeds the permitted use, you will need to obtain permission directly from the copyright holder. To view a copy of this licence, visit http://creativecommons.org/licenses/by$\mathrm{nc} / 4.0 /$.

\section{REFERENCES}

1. Alegre-Sánchez A, Jiménez-Gómez N, Boixeda P. Laser-assisted drug delivery. Actas Dermosifiliogr. 2018;109(10):858-67.

2. Ali FR, Al-Niaimi F. Laser-assisted drug delivery in dermatology: from animal models to clinical practice. Laser Med Sci. 2016;31(2):373-81.

3. Stumpp OF, Bedi VP, Wyatt D, et al. In vivo confocal imaging of epidermal cell migration and dermal changes post nonablative fractional resurfacing: study of the wound healing process with corroborated histopathologic evidence. J Biomed Opt. 2009;14:024018.

4. Waibel JS, Rudnick A, Shagalov DR, Nicolazzo DM. Update of ablative fractionated lasers to enhance cutaneous topical drug delivery. Adv Ther. 2017;34(8):1840-9.

5. Ali FR, Wlodek C, Lear JT. The role of ingenol mebutate in the treatment of actinic keratoses. Dermatol Ther. 2012;2:8.

6. Wlodek C, Ali FR, Lear JT. Use of photodynamic therapy for treatment of actinic keratoses in organ transplant recipients. Biomed Res Int. 2013:349526.

7. Erlendsson AM, Olesen UH, Haedersdal M, Rossi AM. Ablative fractional laser-assisted treatments for keratinocyte carcinomas and its precursors-clinical review and future perspectives. Adv Drug Deliv Rev. 2020;153:185-94. (S0169-409X(20)30003-X)

8. Karmisholt KE, Haedersdal M. Ablative fractional laser intensifies treatment outcome of scalp actinic keratoses with ingenol mebutate: a case report. J Eur Acad Dermatol. 2016;30(5):854-5. 
9. Helsing $\mathrm{P}$, Togsverd-Bo $\mathrm{K}$, Veierød $\mathrm{MB}$, et al. Intensified fractional $\mathrm{CO}_{2}$ laser-assisted photodynamic therapy vs. laser alone for organ transplant recipients with multiple actinic keratoses and wartlike lesions: a randomized half-side comparative trial on dorsal hands. Br J Dermatol. 2013;169: 1087-92.

10. Song HS, Jung S-E, Jang YH, et al. Fractional carbon dioxide laser-assisted photodynamic therapy for patients with actinic keratosis. Photodermatol Photoimmunol Photomed. 2015;31(6):296-301.

11. Kalil CL, Reinehr CP, de Mattos ML, Bakos RM. Combination therapies: laser-assisted delivery of 5-fluorouracil. Dermatol Surg. 2019;45(3):485-7.

12. Micali G, Lacarrubba F, Nasca MR. Reply to: "Laser assisted drug delivery: enhanced response to ingenolmebutate after ablative fractional laser treatment." J Am Acad Dermatol. 2015;72(2):365-6.

13. Kim SK, Park JY, Song HS, Kim YS, Kim YC. Photodynamic therapy with ablative carbon dioxide fractional laser for treating Bowen disease. Ann Dermatol. 2013;25(3):335-9.

14. Ko DY, Kim KH, Song KH. A randomized trial comparing methyl aminolaevulinate photodynamic therapy with and without Er:YAG ablative fractional laser treatment in Asian patients with lower extremity Bowen disease: results from a 12-month follow-up. Br J Dermatol. 2014;170: 165-72.

15. Nguyen BT, Gan SD, Konnikov N, Liang CA. Treatment of superficial basal cell carcinoma and squamous cell carcinoma in situ on the trunk and extremities with ablative fractional laser-assisted delivery of topical fluorouracil. J Am Acad Dermatol. $2015 ; 72: 558-60$.

16. Telfer NR, Colver GB, Morton CA. British Association of Dermatologists guidelines for the management of basal cell carcinoma. Br J Dermatol. 2008;159:35-48.

17. Haedersdal M, Togsverd-Bo K, Paasch U. Case reports on the potential of fractional laser-assisted photodynamic therapy for basal cell carcinomas. Lasers Med Sci. 2012;27:1091-3.

18. Lippert J, Smucler R, Vlk M. Fractional carbon dioxide laser improves nodular basal cell carcinoma treatment with photodynamic therapy with methyl 5-aminolevulinate. Dermatol Surg. 2013;39:1202-8.

19. Haak CS, Togsverd-Bo K, Thaysen-Petersen D, et al. Fractional laser-mediated photodynamic therapy of high-risk basal cell carcinomas-a randomized clinical trial. Br J Dermatol. 2015;172:215-22.
20. Choi SH, Kim KH, Song KH. Efficacy of ablative fractional laser-assisted photodynamic therapy for the treatment of actinic cheilitis: 12-month followup results of a prospective, randomized, comparative trial. Br J Dermatol. 2015;173:184-91.

21. Markopoulos A, Albanidou-Farmaki E, Kayavis I. Actinic cheilitis: clinical and pathologic characteristics in 65 cases. Oral Dis. 2004;10:212-6.

22. Bachhav YG, Heinrich A, Kalia YN. Controlled intra- and transdermal protein delivery using a minimally invasive erbium:YAG fractional laser ablation technology. Eur J Pharm Biopharm. 2013;84:355-64.

23. Forbat E, Al-Niaimi F, Ali FR. The emerging importance of tranexamic acid in dermatology. Clin Exp Dermatol. 2020;45(4):445-9.

24. Wanitphakdeedecha R, Sy-Alvarado F, Patthamalai P, Techapichetvanich T, Eimpunth S, Manuskiatti $W$. The efficacy in treatment of facial melasma with thulium 1927-nm fractional laser-assisted topical tranexamic acid delivery: a split-face, double-blind, randomized controlled pilot study. Lasers Med Sci. 2020;35(9):2015-21.

25. Tawfic SO, Abdel Halim DM, Albarbary A, Abdelhady M. Assessment of combined fractional $\mathrm{CO}_{2}$ and tranexamic acid in melasma treatment. Lasers Surg Med. 2019;51(1):27-33.

26. Mekawy KM, Sadek A, Seddeik Abdel-Hameed AK. Micro-needling versus fractional carbon dioxide laser for delivery of tranexamic acid in the treatment of melasma: a split-face study. J Cosmet Dermatol. 2020 (Epub ahead of print).

27. Nourmohammadi Abadchi S, Fatemi Naeini F, Beheshtian E. Combination of hydroquinone and fractional $\mathrm{CO}_{2}$ laser versus hydroquinone monotherapy in melasma treatment: a randomized, single-blinded, split-face. Clin Trial Ind J Dermatol. 2019;64(2):129-35.

28. Badawi AM, Osman MA. Fractional erbium-doped yttrium aluminum garnet laser-assisted drug delivery of hydroquinone in the treatment of melasma. Clin Cosmet Investig Dermatol. 2018;11:13-20.

29. Mohamed HAR, Nasif GA, Abdel-Azim ES, Ahmed MAF. Comparative study of fractional erbium: YAG laser vs combined therapy with topical steroid as an adjuvant treatment in melasma. J Cosmet Dermatol. $2019 ; 18(2): 517-23$.

30. Lee W-R, Shen S-C, Fang C-L, et al. Skin pretreatment with an Er:YAG laser promotes the transdermal delivery of three narcotic analgesics. Lasers Med Sci. 2007;22:271-8. 
31. Singer AJ, Weeks R, Regev R. Laser-assisted anesthesia reduces the pain of venous cannulation in children and adults: a randomized controlled trial. Acad Emerg Med. 2006;13:623-8.

32. Meesters AA, Bakker MM, de Rie MA, Wolkerstorfer A. Fractional $\mathrm{CO}_{2}$ laser assisted delivery of topical anesthetics: a randomized controlled pilot study. Lasers Surg Med. 2015;48(2):208-11.

33. Yun PL, Tachihara R, Anderson RR. Efficacy of erbium:yttrium-aluminum-garnet laser-assisted delivery of topical anesthetic. J Am Acad Dermatol. 2002;47:542-7.

34. Qu L, Liu A, Zhou L, et al. Clinical and molecular effects on mature burn scars after treatment with a fractional $\mathrm{CO}(2)$ laser. Lasers Surg Med. 2012;44: $517-24$.

35. Ali FR, Forbat E, Al-Niaimi F. Laser treatment of keloid scars. Dermatol Surg. 2017;43(2):318.

36. Waibel JS, Wulkan AJ, Shumaker PR. Treatment of hypertrophic scars using laser and laser assisted corticosteroid delivery. Lasers Surg Med. 2013;45: 135-40.

37. Waibel JS, Wulkan AJ, Rudnick A, Daoud A. Treatment of hypertrophic scars using laser-assisted corticosteroid versus laser-assisted 5-fluorouracil delivery. Dermatol Surg. 2019;45(3):423-30.

38. Waibel JS, Mi QS, Ozog D, et al. Laser-assisted delivery of vitamin $\mathrm{C}$, vitamin $\mathrm{E}$, and ferulic acid formula serum decreases fractional laser postoperative recovery by increased beta fibroblast growth factor expression. Lasers Surg Med. 2016;48(3): 238-44.

39. Cavalié M, Sillard L, Montaudié H, et al. Treatment of keloids with laser-assisted topical steroid delivery: a retrospective study of 23 cases. Dermatol Ther. 2015;28:74-8.

40. Park JH, Chun JY, Lee JH. Laser-assisted topical corticosteroid delivery for the treatment of keloids. Lasers Med Sci. 2017;32:601-8.

41. Kraeva E, Ho D, Jagdeo J. Successful treatment of keloid with fractionated carbon dioxide $\left(\mathrm{CO}_{2}\right)$ laser and laser-assisted drug delivery of triamcinolone acetonide ointment in an African-American man. J Drugs Dermatol. 2017;16(9):925-7.

42. Rkein A, Ozog D, Waibel JS. Treatment of atrophic scars with fractionated $\mathrm{CO}_{2}$ laser facilitating delivery of topically applied poly-L-lactic acid. Dermatol Surg. 2014;40:624-31.

43. Trelles MA, Leclère FM, Martínez-Carpio PA. Fractional carbon dioxide laser and acoustic-pressure ultrasound for transepidermal delivery of cosmeceuticals: a novel method of facial rejuvenation. Aesthetic Plast Surg. 2013;37:965-72.

44. Mahmoud BH, Burnett C, Ozog D. Prospective randomized controlled study to determine the effect of topical application of botulinum toxin A for crow's feet after treatment with ablative fractional $\mathrm{CO}_{2}$ laser. Dermatol Surg. 2015;41:S75-81.

45. Brandt F, O'Connell C, Cazzaniga A, Waugh JM. Efficacy and safety evaluation of a novel botulinum toxin topical gel for the treatment of moderate to severe lateral canthal lines. Dermatol Surg. 2010;36: 2111-8.

46. Bertin AC, Vilarinho A, Junqueira AL. Fractional non-ablative laser-assisted drug delivery leads to improvement in male and female pattern hair loss. J Cosmet Laser Ther. 2018;20(7-8):391-4.

47. Cohen PR. Laser-assisted drug delivery for the treatment of androgenetic alopecia: ablative laser fractional photothermolysis to enhance cutaneous topical delivery of platelet-rich plasma-with or without concurrent bimatoprost and/or minoxidil. Dermatol Online J. 2019;25(2).

48. Majid I, Jeelani S, Imran S. Fractional carbon dioxide laser in combination with topical corticosteroid application in resistant alopecia areata: a case series. J Cutan Aesthet Surg. 2018;11(4):217-21.

49. Salah M, Samy N, Fawzy MM, Farrag AR, Shehata H, Hany A. The effect of the fractional carbon dioxide laser on improving minoxidil delivery for the treatment of androgenetic alopecia. J Lasers Med Sci. 2020;11(1):29-36.

50. Sobhi RM, Sharaoui I, El Nabarawy EA, Esmail RS, Hegazy RA, Aref DH. Comparative study of fractional $\mathrm{CO}_{2}$ laser and fractional $\mathrm{CO}_{2}$ laser-assisted drug delivery of topical steroid and topical vitamin $C$ in macular amyloidosis. Lasers Med Sci. 2018;33(4):909-16.

51. Chen X, Wang J, Shah D, Wu MX. An update on the use of laser technology in skin vaccination. Expert Rev Vaccines. 2013;12:1313-23.

52. Ma G, Wu P, Lin X, et al. Fractional carbon dioxide laser-assisted drug delivery of topical timolol solution for the treatment of deep infantile hemangioma: a pilot study. Pediatr Dermatol. 2014;31: 286-91.

53. Sun L, Wang C, Cao Y, et al. Fractional 2940-nm Er: YAG laser-assisted drug delivery of timolol maleate for the treatment of deep infantile hemangioma. J Dermatol Treat. 2020;22:1-7. 
54. Yoo KH, Kim BJ, Kim MN. Enhanced efficacy of photodynamic therapy with methyl 5-aminolevulinic acid in recalcitrant periungual warts after ablative carbon dioxide fractional laser: a pilot study. Dermatol Surg. 2009;35:1927-32.

55. Coates M, Patel J, Powers C, Burton C. Laser-assisted delivery of topical cidofovir in the treatment of plantar warts. J Drugs Dermatol. 2019;18(7):663-5.

56. Junsuwan N, Manuskiatti W, Phothong W, Wanitphakdeedecha R. Fractional $\mathrm{CO}_{2}$ laser-assisted botulinum toxin type A delivery for the treatment of primary palmar hyperhidrosis. Lasers Med Sci. 2020 (Epub ahead of print).

57. Zaleski-Larsen LA, Fabi SG. Laser-assisted drug delivery. Dermatol Surg. 2016;42(8):919-31.
58. Ibrahim O, Wenande E, Hogan S, Arndt KA, Haedersdal M, Dover JS. Challenges to laser-assisted drug delivery: applying theory to clinical practice. Lasers Surg Med. 2018;50(1):20-7.

59. Haedersdal M, Erlendsson AM, Paasch U, Anderson RR. Translational medicine in the field of ablative fractional laser (AFXL)-assisted drug delivery: a critical review from basics to current clinical status. J Am Acad Dermatol. 2016;74(5):981-1004.

60. Wenande E, Erlendsson AM, Haedersdal M. Opportunities for laser-assisted drug delivery in the treatment of cutaneous disorders. Semin Cutan Med Surg. 2017;36(4):192-201. 